Swarthmore College

Works

1995

\title{
Optical Measurements On The BP III To Isotropic Phase Transition In Highly Chiral Liquid Crystals
}

Jonathan Brent Becker , '92

Peter J. Collings

Swarthmore College, pcollin1@swarthmore.edu

Follow this and additional works at: https://works.swarthmore.edu/fac-physics

Part of the Physics Commons

Let us know how access to these works benefits you

\section{Recommended Citation}

Jonathan Brent Becker, '92 and Peter J. Collings. (1995). "Optical Measurements On The BP III To Isotropic Phase Transition In Highly Chiral Liquid Crystals". Molecular Crystals And Liquid Crystals. Volume 265, Issue 1. 163-170. DOI: 10.1080/10587259508041688

https://works.swarthmore.edu/fac-physics/227

This work is brought to you for free by Swarthmore College Libraries' Works. It has been accepted for inclusion in Physics \& Astronomy Faculty Works by an authorized administrator of Works. For more information, please contact myworks@swarthmore.edu. 
Mol. Cryst. Liq. Cryst. 1995, Vol. 265, pp. 163-170

Reprinis available directly from the publisher

photocopying permitted by license only
- 1995 OPA (Overseas Publishers Association) Amsterdam B.V. Published under license by Gordon and Breach Science Publishers SA Printed in Malaysia

OPTICAL MEASUREMENTS ON THE BP III TO ISOTROPIC PHASE TRANSITION IN HIGHLY CHIRAL LIQUID CRYSTALS

JONATHAN B. BECKER AND PETER J. COLLINGS

Department of Physics \& Astronomy, Swarthmore College, Swarthmore, PA 19081, U.S.A.

Abstract Optical activity and light scattering measurements in the chiral-racemic system of 4-12methylbutyl)phenyl-4-hexyloxybenzoate (CE4) reveal a much larger decrease in the discontinuity at the third blue phase (BP III) to isotropic phase transition than reported previously using adiabatic scanning calorimetry on another compound. Whereas these new data show that the transition is still slightly discontinuous for the pure optical isomer of CE4, similar light scattering measurements on the pure optical isomer of 4"-(2methylbutylphenyl) -4' - (2-methylbutyl) -4-biphenylcarboxylate (CE2), which is more chiral than CE4, show no discontinuity at all.

\section{INTRODUCTION}

Recent experimental results point out that the transition to the isotropic phase in highly chiral liquid crystals is very different from the isotropic transition in low chirality or non-chiral liquid crystals. These measurements include optical activity and light scattering experiments (see Ref. 1 for a recent review) and thermodynamic experiments.2,3 one difference stems from the fact that the transition to the isotropic phase in highly chiral systems is from a defect mediated phase, either the third blue phase or the smectic A* phase, rather than from a phase without intrinsic defects. While the smectic $A^{*}$ phase can be understood as a twist grain boundary phase, 4 the third blue phase is not well understood at a11. Regardless of which of these two phases occurs below the isotropic phase, the short-range fluctuations in the isotropic phase of highly chiral liquid crystals are quite large and possess a temperature dependence which departs from mean-field theory. 1 
There is also experimental evidence that the transition from the third blue phase (BP III) to the isotropic phase becomes less discontinuous as the chirality is increased. ${ }^{3,5}$ While this general behavior is predicted by mean-field theory, 6,7 theoretical techniques which take into account short-range fluctuations are going to be necessary to understand this transition when the discontinuity becomes very small. The adiabatic scanning calorimetry experiments of Voets and Van Dael on $\mathrm{CE}^{3}$ clearly show a decrease in the latent heat at the isotropic transition with increasing chirality. An even larger decrease was seen by these workers in the more chiral CE4. ${ }^{8}$ In order to provide optical evidence for this decrease in the system of highest chirality studied by Voets and Van Dael, we conducted precise optical activity and light scattering measurements on 4-(2methylbutyl)phenyl-4-hexyloxybenzoate (CE4). By achieving temperature homogeneity and temperature precision of less than $5 \mathrm{mK}$, we were able to measure small discontinuities. By performing the experiments on the chiral-racemic system (CE4), we were able to vary the chirality of the sample while keeping all other thermodynamic parameters constant. Our results are very similar to those of Voets and Van Dael, ${ }^{3}$ in that the discontinuities in both the optical activity and light scattering intensity decrease fairly linearly with an increase in chirality. Unlike the results for CE6 where the latent heat decreases by about a factor of two as the chiral fraction changes from 0 to 1 , the discontinuities in the optical properties decrease by roughly an order of magnitude in $\mathrm{CE} 4$. An additional light scattering experiment on the pure optical isomer of $4^{\prime \prime}-12-$ methylbutylphenyl)-4'-(2-methylbutyl)-4-biphenyl-carboxylate (CE2), which is more chiral than CE4, shows no discontinuity at the BP III to isotropic transition.

\section{THEORY}

When a chiral term is added to the Landau-deGennes free 
energy, interesting new effects occur in the vicinity of the isotropic transition. When the chirality term is small, the transition is from the chiral nematic phase to the isotropic phase and the latent heat is similar to that of a nematic to isotropic phase transition. As the coefficient in front of the chiral term is increased, however, new phases become stable near the isotropic phase transition (the blue phases) and the latent heat of the transition to the isotropic phase decreases. ${ }^{6,7}$ This theory predicts that the transition from the chiral nematic to isotropic phase in fact becomes continuous at high chirality. This situation is never realized, however, since the transition to the isotropic phase at high chirality is always from a blue phase and not from the chiral nematic phase. Very recent work by Keyes ${ }^{9}$ suggests that coupling between the fluctuating chiral modes in highly chiral systems causes the BP III - isotropic transition line to end at a critical point as the chirality is increased. If this is correct, there should be no discontinuities at all at the isotropic transition if the chirality is high enough.

\section{EXPERIMENT}

Chiral CE4 and racemic CE4R were obtained from BDH and used without further purification. Mixtures of various chiral fractions were made and placed between two pieces of glass separated by a $125 \mu \mathrm{m}$ mylar spacer. These samples were then placed in an Instec MKl heating stage, and both optical activity and light scattering experiments were performed at temperatures separated by $5 \mathrm{mK}$. Since phase transitions completely took place between two data points, the temperature homogeneity and the temperature control precision must have been less than $5 \mathrm{mK}$.

The optical activity apparatus has been described previously.10 It is capable of measuring rotation angles to $\pm 0.001^{\circ}$, which is necessary for measurements on thin samples in the isotropic phase. The wavelength of light used was 633 
nm. Measurements were obtained for temperatures within $0.1 \mathrm{~K}$ on either side of the BP III to isotropic transition for samples with chiral fractions between 0.8 and 1 .

The light scattering apparatus has also been described previously.11 Right circular polarized light was incident on the sample and only right circularly polarized light was detected. The scattering angle was close enough to $180^{\circ}$ so that only the dominant chiral mode, in this case the $m=-2$ mode, contributed significantly to the scattering. The wavelength of the light was $633 \mathrm{~nm}$. Again, measurements were made within a $0.1 \mathrm{~K}$ interval on either side of the BP III to isotropic transition for samples with chiral fractions between 0.85 and 1 . A similar experiment was performed using the pure optical isomer of $\mathrm{CE} 2$ in a region roughly $1 \mathrm{~K}$ on either side of the BP III to isotropic transition. In this case, the data were obtained at $25 \mathrm{mK}$ intervals.

\section{RESULTS}

Fig. 1 displays the optical activity data for 4 different chiral fractions. Both the decrease in the discontinuity at the transition and the increase in pretransitional optical activity as the chirality increases are evident. In fact, one might be tempted to say that the optical activity is continuous across the BP III to isotropic transition in the sample with chiral fraction equal to 1 .

The light scattering data are displayed in Fig. 2 . Again, the decrease in the discontinuity and the increase in pretransitional light scattering are clearly evident as the chiral fraction increases. The magnitude of the discontinuity can be determined by fitting a straight line to the BP III data and extrapolating it to temperatures in the isotropic phase. The difference between this 1 ine and the actual intensity at the first data point in the isotropic phase can then be used to represent the magnitude of the discontinuity. A graph of the discontinuity measured in this fashion versus chiral fraction is shown in Fig. 3. The error 
bars represent the uncertainty in determining the first point in the isotropic phase. The decrease in this discontinuity with increasing chiral fraction is clearly evident, and a straight line fitted to the points extrapolates to zero discontinuity at a chiral fraction between 1.05 and 1.10 .
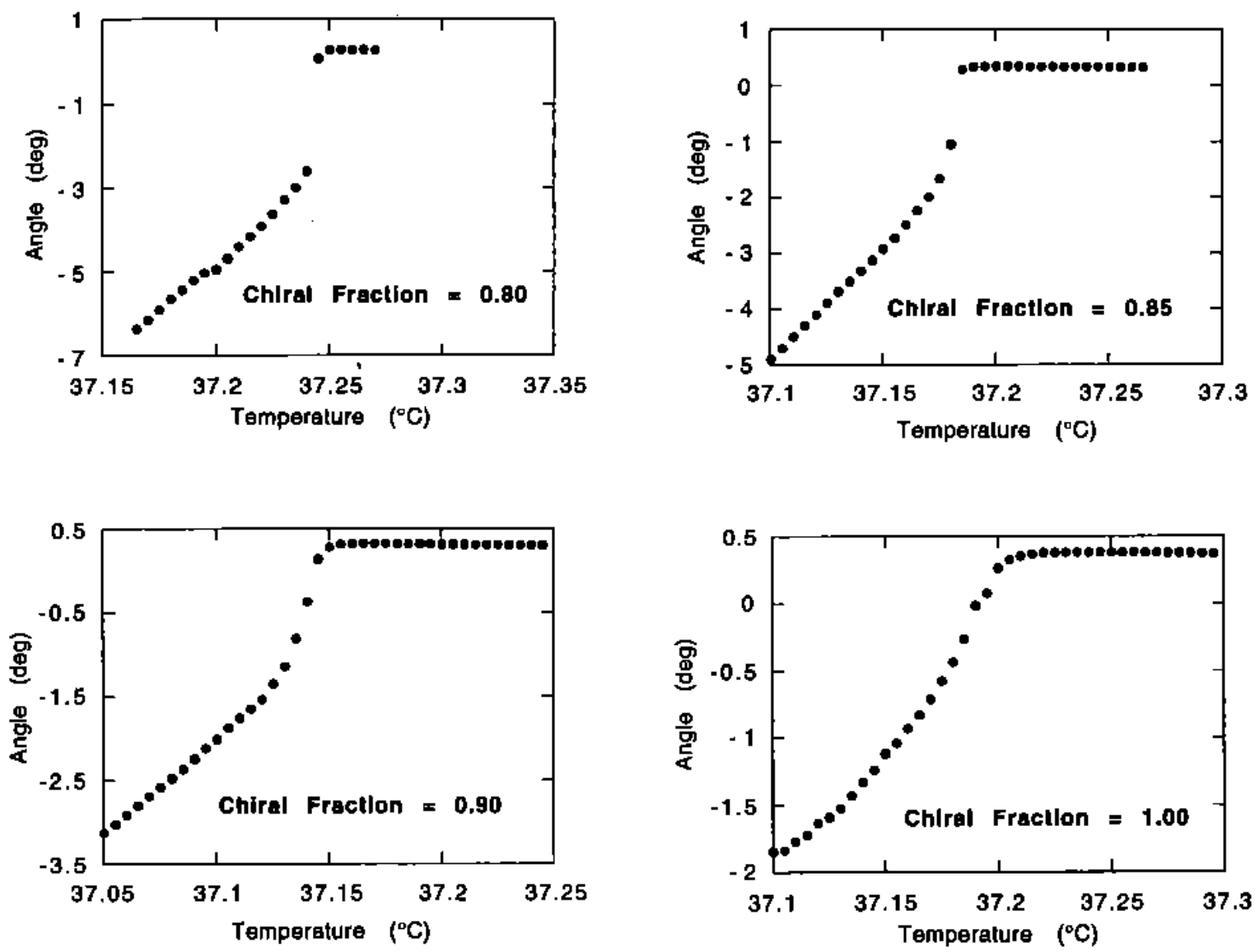

FIGURE 1 Optical activity of CE4 (BP III - Isotropic).

The data for the pure chiral isomer of CE2 are shown in Fig. 4. Prior measurements on CE2 demonstrate that the location of the BP III to isotropic transition is very difficult to determine optically, because the transition appears to be continuous. Discontinuities in the slope of both optical activity ${ }^{12}$ and light scattering intensity ${ }^{10}$ versus temperature are present and consistently place the transition $1.5 \mathrm{~K}$ above the BP I to BP III transition (which is optically discontinuous). Notice that no discontinuity is apparent in this region of Fig. 3. Even the change in slope, which is clear when data are taken over a wider region in the isotropic phase, is not clearly evident in this new data. 

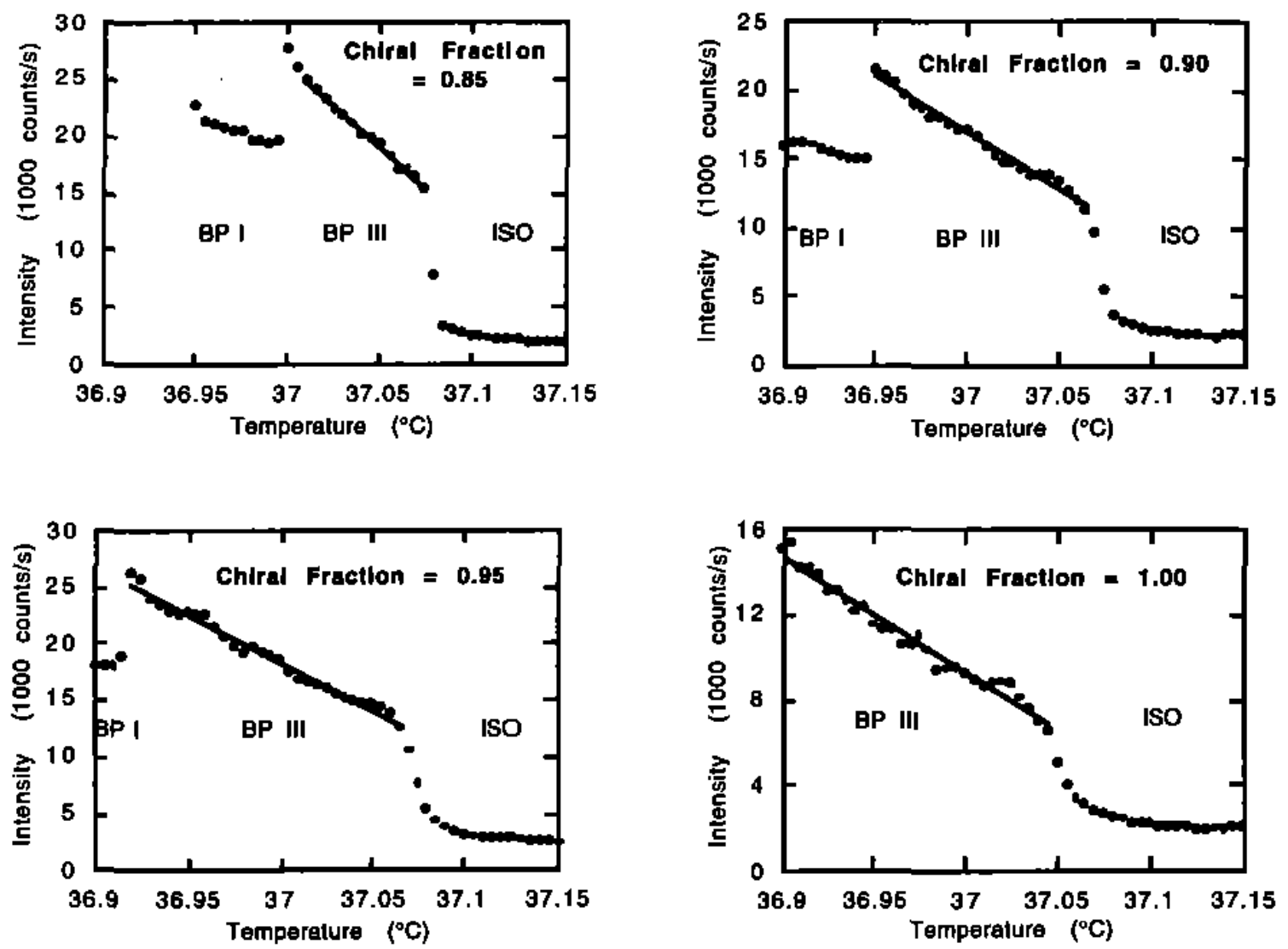

FIGURE 2 Light scattering for CE4. The lines are 1 inear least square fits to the BP III data.

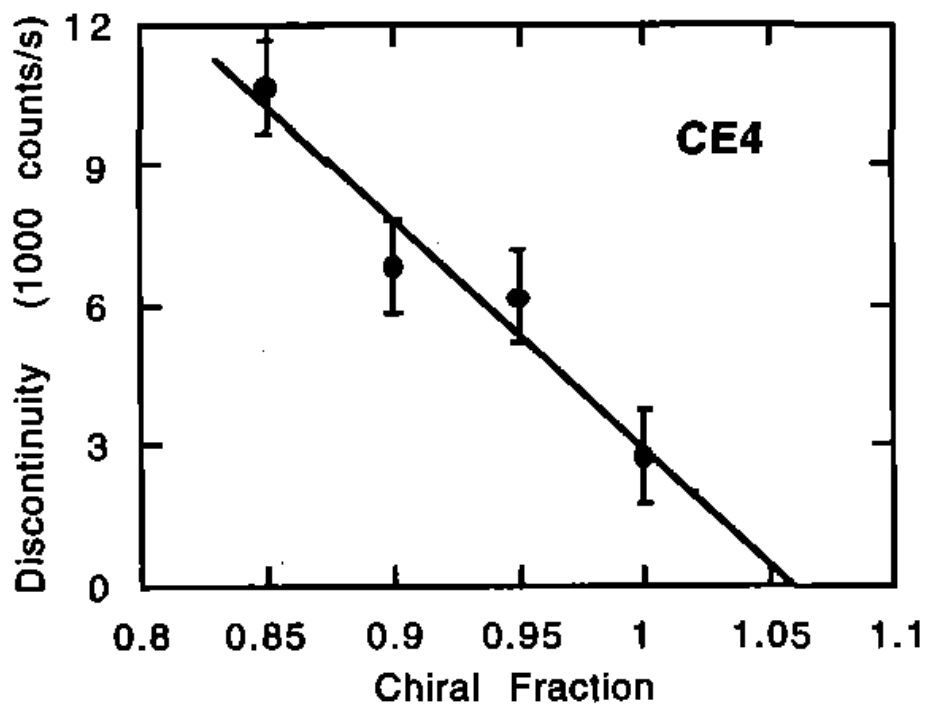

FIGURE 3 Discontinuity in the light scattering intensity of CE4 at the BP III - isotropic phase transition. 


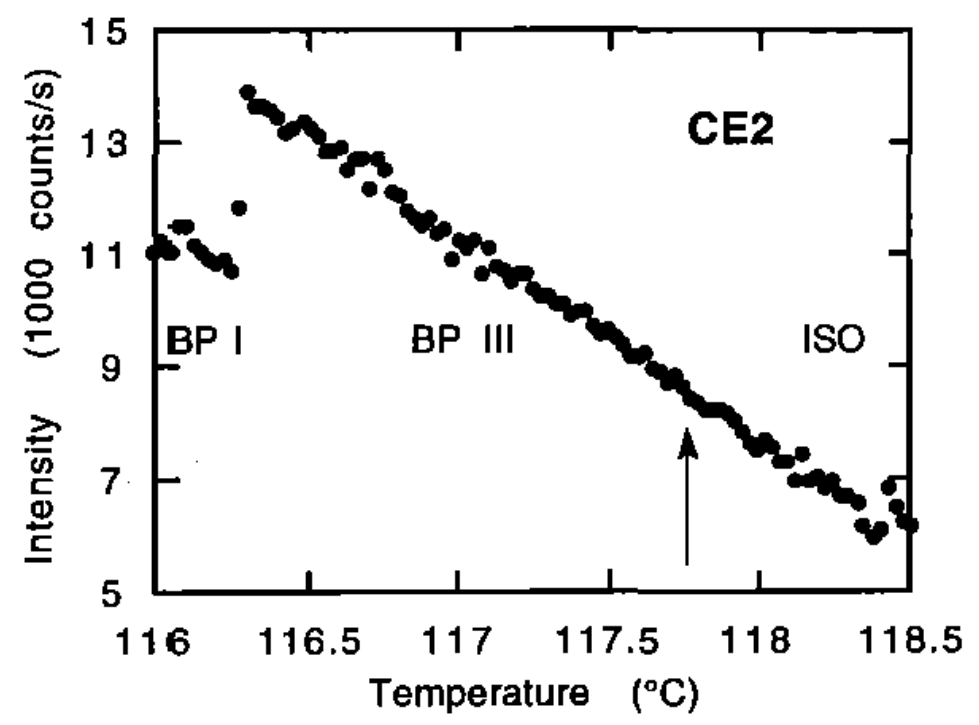

FIGURE 4 Light Scattering Intensity for CE2. The arrow marks the BP III - isotropic phase transition.

\section{DISCUSSION}

The optical results reported here provide strong evidence that the BP III to isotropic transition can become nearly continuous or perhaps completely continuous. Whereas prior results on $\mathrm{CE} 6$ demonstrated a reduction in the latent heat by a factor of about two, extrapolating the line in Fig. 3 back to zero chiral fraction makes the reduction in the discontinuity at the BP III to isotropic transition nearly a factor of ten. When this large reduction is combined with the results presented here on $\mathrm{CE} 2$, it provides ample justification for continued experiments on highly chiral liquid crystals.

Clearly a chiral-racemic system with the chirality of CE2 is required for the measurements necessary to ascertain if high chirality can produce a continuous transition. Other experimental probes such as dynamic light scattering should also provide important information concerning the dynamics of this phase transition.

If a critical point of some type is present, the most pressing question will be whether it is a critical end point 
or a tricritical point. This is an open question theoretically, since the order parameter (and therefore the symmetry of the order parameter) for BP III is not known. Dynamical information on the fluctuations near this transition may be useful in determining the nature of the critical point.

\section{ACKNOWLEDGEMENTS}

These results are based on work supported by the National Science Foundation under Grants Nos. DMR-9196048 and DMR9224617 and the W. M. Keck Foundation. Useful discussions with P. Crooker, R. Hornreich, P. Keyes, T. Lubensky, and W. Van Dael are gratefully acknowledged.

\section{REFERENCES}

1. P. J. Collings, Mod. Phys. Lett., 6, 425 (1992).

2. J. Thoen, Phys, Rev, A, 37, 1754 (1988).

3. G. Voets and W. Van Dael, Liq. Cryst., 14, 617 (1993).

4. J. W. Goody, M, A. Waugh, S. M. Stein, E. Chin, R. Pindak, and J. S. Patel, J.Am. Chem. SoC., 111, 8119 (1989) .

5. P. R. Battle, J. D. Miller, and P. J. Collings, Phys. Rev, A, 36, 369 (1987).

6. S. A. Brazovskii and S. G. Dmitriev, Zh. Eksp. Teor. Fiz, 69, 979 (1975) [Sov. Phys. JETP, 42, 497 (1975); . A. Brazovskii and V. M. Filev, Zh. Eksp. Teor. Fiz., 75, 1140 (1978) [Sov. Phys. JETP, 48, 573 (1978)].

7. H. Grebel, R. M. Hornreich, and S. Shtrikman, Phys.Rev. A, 28, 1114 (1983); Phys. Rev. A, 30, 3264 (1984).

8. G. Voets and $W$. Van Dael, private communication.

9. P. H. Keyes, private communication.

10. J. D. Rosenzweig and P. J. Collings, Phys Rey. E, $\underline{47}$, 1876 (1993).

11. J. E. Wyse and P. J. Collings, Phys.Rev. A, $\underline{45}, 2449$ (1992).

12. J. Ennis, J. E. Wyse, and P. J. Collings, Lio. Cryst., $\underline{\text {, }}$ 861 (1989). 